\title{
Chemical Factors which Prompt Oral Pathological Phenomena In Some Nutrition Diseases
}

\author{
RAOUL VASILE LUPUSORU1', GABRIEL TOPOR ${ }^{2 *}$, INGRITH CRENGUTA MIRON ${ }^{1 *}$, MIHAELA GRIGORE ${ }^{1}$, IRINA ESANU ${ }^{1}$ \\ ${ }^{1}$ Grigore T. Popa University of Medicine and Pharmacy, 16 Universitatii Str., 700115, lasi, Romania \\ 2Dunarea de J os University of Galati, Faculty Of Medicine And Pharmacy, 47 Domneasca Str., 800008, Galati, Romania
}

In so-called general pathology, the centre of gravity must fall precisely on the experimental pathology, on the analysis of experimental pathological processes. To understand the life of a diseased organism, it is necessary to know the morphological changes at different stages of development of the pathological processes. Morphopathology deals with the study of morphological changes in a diseased organism. Both disciplines, namely both pathophysiology, as well as morphopathology, complement each other and together constitute a vast field of medical knowledge, namely the so-called pathology (pathos = suffering, disease, and logos = science). Physiopathology uses the data of the morphological changes found as a result of the pathological processes and it is closely related to the pathological anatomy, which deals with the same problems, but from another point of view: both have the same object of study, namely, the diseased human organism. Any pathological action provokes from the very beginning a defence of the body, directed against the injurious agent or the lesion. Pathological phenomena are recognized not only by studying the functional changes of the body and its various parts; taking into account the unity between function and form, it is necessary to study the morphological changes. From the research carried the following resulted: from the total of 165 cases, 102 cases (61.81\%) presented diabetes, 34 cases (20.62\%) obesity, 29 cases (17.57\%) gout. In the treatment of oral mucosal lesions, strong antiseptics will be avoided since these are necrotizing (multiple cases of diabetic angiopathy are known), attention should also be paid to performing fixed or mobile prosthetic works that do not have to traumatize the periodontium and the mucosa of the prosthetic field in order to avoid the possibility of over-infection. Avoid bleeding manoeuvres in case of a diabetic patient with high blood sugar, taking into account the risk of diabetic coma.

Key words: general pathology, functional changes, changes in metabolism, nutrition.

Any pathological process is accompanied by quantitative and qualitative changes of metabolism, which is the main form of life manifestation. The metabolic disorders occur after the central nervous system activity is affected.

Metabolic diseases are disorders affecting a number of nutrients, often characterized by abnormal chemical reactions that may result in various changes in the metabolic process. These conditions have on the one hand hereditary factors (some can be detected from birth), but they can also be caused by lifelong illnesses (identified after the appearance of specific symptoms).

The totality of disorders that arise on various metabolic levels of food principles are grouped and are called metabolic diseases.

Diabetes mellitusis par excellence a metabolic disorder initially affecting the carbohydrate, lipidic and protidic secondary metabolism; it is a genetically determined metabolic disorder, which in its complete clinical expression is characterized by hyperglycemia a jeun, atherosclerotic vascular disease, microangiopathy and neuropathy; it is a genetically determined metabolic disease with chronic and staged evolution, influenced by various etiological factors characterized by disorders of carbohydrate metabolism, followed by disorders of lipid and protidic metabolism, angiopathy and neuropathy being component elements of the disease [1-3].

Acute complications are a consequence of metabolic decompensation induced by insulin deficiency associated with or not to the increased secretion of one or more counter-regulating hormones, favouring increased liver

*email: gabriel.topor@yahoo.com; lucmir@gmail.com production and mobilization of peripheral energetic substances.

Classical diabetes occurs through the symptomatic triad: polyuria, polydipsia, polyphagia. Although large amounts of glucose are present in the blood, tissues cannot use the glucose, and thus it is eliminated through urine. Polyuria occurs early, usually in $65-70 \%$ of cases. Polyphagia occurs as a compensatory effect, replacing losses in food principles. During the polyphagia, progressive weight loss and physical asthenia occur. The diabetic patient lacking glucose for energy acquisition uses albumin and fat, the burning of which produces ketones that induce acidosis. The ketone bodies are eliminated through urine and breath in the form of acetone, a substance that has an aromatic sower aroma (of rotted apple) [4-6].

The loss of salt through the urine is getting worse because the kidney eliminates the ketone bodies combined with sodium, the main constituent of the salt. Lacking the necessary liquids, intoxicated with ketone bodies, the diabetic enters the coma losing consciousness.

Diabetic microangiopathy is a condition of the vascular terminal package (arterioles, capillaries, venules) with a preferential location in the kidney, retina or vasorum vessels.

Microangiopathy is found in all stages of diabetes: it is established early and is present especially in the stages of latent diabetes; it is considered a symptom rather than complication; it is especially common in young patients [7-9].

Diabetes is a disorder in glucose metabolism that leads to hyperglycemia and glycosuria. Due to the interconversion of carbohydrate, lipid and protein metabolism in the Krebs cycle, the last two metabolisms 
are affected, which explains the serious symptoms of diabetes. In glucose metabolism we distinguish three main processes: glycogenogenesis - glucose deposition in glycogen at the liver level; glucogenolysis - glycogen transformation into glucose by hydrolysis and its mobilization in the blood based on the gluconeogenesis needs, namely glucose formation from lipids and protides in the Krebs cycle. The prolonged progression of symptoms characteristic of the pathology causes important organ damage, up to fine structure modification, and acute or chronic complications are of particularly serious severity for diabetic patients because they reduce the duration and quality of life and the patient's behaviour reflects changed aspects in his lifestyle [10,11].

Gingival biopsies in cases of latent diabetes show intense capillary congestion and sometimes a capillary neoformation the diameter of which does not exceed that of the hematite. The capillaries are rectilinear and their walls are rigid due to fibrosis. The alveolar bone resorbs more quickly in case of diabetics. In the sub-mucous area a capillary neovascularization is found, favoured by hypoxia. Besides these manifestations which are characteristic to diabetes, there are also oro-dental manifestations.

Oral cavity lesions are polymorphic and are physiologically interpreted very differently; oro-dental changes in diabetes have degenerative arterial involvement due to early tissue aging. Oral dental changes are caused by disturbances of dental tissue mineralization in the form of adamantine hyperplasias, adamantine erosions. The carious process is not frequent, pre-existing cavities get worse, having a hypomineralization of hard dental tissues. Accelerated teeth loss in diabetes is due to pathological mobility rather than to dental caries [12].

There is no correlation between diabetes mellitus and dental caries. Teeth without caries exhibit pulp modifications; in case of damaged teeth the pulp exhibits acute inflammatory tendencies with frequent pulp abscesses and retrograde pulp necrosis.

The cement shows changes in its structure, with fine limestone being found. Regarding saliva, diabetic patients are faced with a decrease in salivary flow, explained by diabetic polyuria or salivary gland hypofunction following a diffuse process of glandular sclerosis. Saliva flow dynamics produces some lesions located in the oral cavity: cracked lips with bleeding crusts, jaw mucosa is dry, lacquered; the tongue is red, cracked, depapillated, bulky.

Persistent dryness of the mouth causes a halitosis with a raw apple smell. Tartar and dental plaque deposits are common. Parotid hypertrophy is common in diabetes. Parotid hypertrophy is the result of hypersecretion of antiinsulin hormone, producing the same disorders as insular failure by blocking insulin action [13,14].

Diabetic gingivitis concerns only the periodontium of the cover, without including the alveolar ligaments and the alveolar bone.

Chronic superficial periodontitis is very common in younger age, considered as an early manifestation of the disease. In deep chronic marginal periodontitis, the patient has masticatory embarrassment, tooth mobility, fetid halitosis, purple appearance of the gum due to stasis, tearing of the gums' papillae from the teeth, purulent secretion, dental mobility.

Periodontitis is a dystrophic-atrophic form of chronic marginal periodontitis without inflammatory phenomena, gingival retraction. Parodontopathies in diabetes begin early, evolve rapidly and lead to partial or total editions.

Hyperglycaemia is the expression of decreased intracellular glycolysis. It can lead to the periodontal condition due to the reduction or inactivation of insulin; it is responsible for the tendency to chronic infections in periodontium. Diabetes creates in the alveolar bone favourable conditions for the internal development of the alveolar pyorrhea in the form of an osteoporotic atrophy process $[15,16]$.

Low body resistance determines periodontal complications such as gingival-periodontal abscesses.

Diabetic stomatitis is characterized by dry mouth sensation, gingival pruritus, oral pyrexia, gingivorrhagia, stings and pruritus at the anterior $1 / 3$ level of the tongue, acetone smell. The oral mucosa is bright red, with gingival ulcerated edges, interdental papillae increased in volume and bleeds easily. Ulcero-necrotic stomatitis may worsen the disease, but diabetes can also cause ulcero-necrotic stomatitis.

Another manifestation encountered in the diabetic patient is the cheilitis characterized by dry lips covered with squamous areas, with commissural rhagas. There is an increased frequency of glossopathy, in the sense that the tongue is enlarged by volume, grooved by trenches, hyperemiated fusiform papillae. In other cases, the tongue is flaccid, atonic, with pain, burns, tingling and pricking. We can also find gangrene injuries. Jugular mucosa leucoplakias have the appearance of a white-grey patch; dental and abundant tartar, alveolar crest atrophy are other manifestations encountered. Post-surgical haemorrhages are also encountered due to the increase of vascular permeability and fragility $[17,18]$.

The trigeminal neuralgia encountered in diabetes patients has the following characteristics: migrating pain, bilateral pain; night exacerbation; disappears after administration of insulin.

Sometimes we can also find thrushes, mycotic stomatitis, candida albicans, etc. Because of vasomotor disorders and changes in arterial walls, we can find retrograde pulp necrosis, which is especially common in the elderly. Inflammation of the oral mucous membrane could be explained by the insufficient oxidation of carbohydrates and absorption of lipids, by the diminishing of cell osmotic power, by the lowering of alkalinity and increase in the percentage of carbohydrates. The severity of oral mucosal lesions is not proportional to glycemia or glycosuria levels. The decrease in body resistance predisposes to the occurrence of some complications in the form of gingival-periodontal abscesses.

In obesity, nutrition disease, metabolic disease, it has the following general manifestations: hyperlipogenesis, weight gain over normal limits; very rich fatty mass.

The disease develops in three stages: 1- neomogenous and endocrinopathy manifest stage, 2- the stage of metabolic complications (diabetogen and atherogen); 3visceralization '! cardiovascular visceropathy and renal visceropathy stage $[19,20]$.

From the point of view of the oral-dental manifestations it presents: hypersecretion of salivary glands ( sialorrhea, ptyalism); hyperorexia, bitter taste, enlarged, smooth, sometimes depapillated tongue; glossitis; partial edentation; periodontitis; abundant dental tartar; I-III degree dental mobility; dentinal hyperaesthesia, marginal periodontitis, oral mucosal atrophy; erythematous stomatitis; simple caries; angular cheilitis.

\section{Oral syndrome in gout}

Gout is a metabolic entity characterized by a positive uric acid balance, and clinically by specific acute or chronic articular manifestations, as well as by visceral lesions, particularly renal. 
It is a complex disorder of protein metabolism, associated with alteration of lipid and carbohydrate metabolism.

The disease has been known since ancient times, by different names, the last term being that of gout- like drop by drop to the various parts of the body causing their acute illness.

It is the illness of the good living, being found in the past at the wealthy strata [21, 22].

More than half of the patients have the metatarsalphalanx joint of the haluce damaged. The ebb is usually sudden, most frequently nocturnal. Fever is preceded by chills. In chronic form, gout tophi are almost characteristic. All these symptoms are a major clinical manifestation of purine metabolism, which leads to an increase in the amount of uric acid in the body (over $7 \mathrm{mg} \%$ in serum).

\section{Oral syndrome in avitaminosis \\ Avitaminosis A}

Avitaminosis $A$ is manifested by ocular and cutaneous disorders. Xerophthalmia is the characteristic disorder of vitamin A deficiency. Ocular symptoms consist of a conjuncture, then conjunctive xerosis with the danger of keratomalacia. To all these, acute eye infections may be added. Bitot stains are bilateral conjunctival lesions with a white or grey coloration.

Hemeralopia or night cecity may occur in advanced forms. On the skin, keratosis papules appear, especially on the anterior-lateral side of the thighs and on the posterolateral side of the arms. The hair becomes dry, brittle, nails have transversal streaks and the skin is dry. In the digestive tract, diarrhea and achlorhydria occur. Blood carotenoids oscillate between 75 and 150 micrograms\%.

As for the oral manifestations in avitaminosis A, we will encounter: the massive keratinization of the lip and gum submucosa, the red of the lips loses its suppleness, the colour turns to a greyish-blue hue, and the gum has a tendency to hyperplasia. The stenosis extends to the entire oral mucosa and also affects the secretory epithelium of the salivary glands with xerostomia installation; leukoplakia, enamel organ atrophy, white-washed coloured teeth, incisor eruption retardation, jaw hypocalcification, rabbit lip, blue-grey lip coloration, mucoid-sticky saliva, parotid swelling [23, 24].

\section{Avitaminosis $\mathrm{C}=$ scurvy (oral ulcer).}

Vitamin $C$ plays a role in maintaining intercellular substance integrity and formation, in hemopoiesis, in iron absorption, in lipid, carbohydrates metabolism and in the activity of some enzymes (catalase, cathepsin). It has antitoxic, anti-infectious, anti-cataract action.

Clinical symptomatology of avitaminosis C: asthenia; malaise; inability to make physical and intellectual efforts; depression, rheumatic pain; paleness and dryness of the skin; gum disorders.

Oral manifestations are: angular stomatitis that first manifests in the form of a pallor, followed by skin maceration and transverse cracking; cutaneous-mucosal lesions that can touch the wings of the nose, the malarious prolapses, the forehead and the region near the ear; lesions of the lips, of oral mucosa, and of the tongue that are painful, giving a burning sensation, embarrassing the tongue during movement, or when swallowing food and opening the mouth.

Pain and burning in the tongue, cheilitis, angular stomatitis, glossitis with pavement appearance, gingivitis with edema and ulcerations are the main symptoms of avitaminosis B

\section{Avitaminosis B1}

Avitaminosis B1 = Beriberi disease (Indian expression). Vitamin $B 1$ is an active factor in the metabolism of hydrocarbons, being the co-ferment of carboxylase that interferes with the metabolism of pyruvic acid. The vitamin B1 deficiency leads to an increase in the pyruvic acid, lactic acid and to the production of neuritis, polyuria, neuralgia. The body needs $1.5-2 \mathrm{mg} /$ day.

Oral manifestations include oral mucosa vesicles, multiple caries, the stopping of dental eruption, dental dysplasia, enamel and dentin dystrophy. We can also find dry lips with rhagas, burning sensations throughout the oral cavity, red oral mucosa, gingivorrhagia, punctuated, engraved, painful tongue, ulcerous-membranous stomatitis $[25,26]$.

\section{Experimental part}

Material and method

The research resulted in the following: out of the total of 165 cases, 102 cases (61.81\%) presented diabetes, 34 cases $(20.62 \%)$ obesity, 29 cases ( $17.57 \%)$ gout. The cases studied were represented by 112 women $(67.87 \%)$ and 53 males (32.12\%).

The frequency of disorders has increased after 40 years, and the highest number of cases has occurred between 50 and 60 years.

Oro-dental changes have been frequently encountered in diabetic and obese patients in much greater proportions than in healthy controls or with other diseases.

\section{Results and discussions}

Following the study made on 165 patients with orodental manifestations, the following findings were made: anamnesis, research of personal and heredo-collateral history are mandatory stages that every dentist must undergo in front of susceptible, oro-dental lesions, supplemented by laboratory exploration; re-balancing the diabetic is an indispensable thing for local treatment, local anaesthesia with adrenaline is contraindicated, being hyperglycaemic and necrotizing; salivary flow disorders are present in over $90 \%$; oral mucosa exhibits more or less characteristic changes: erythematous stomatitis 13\%, ulcerative-necrotic stomatitis $15 \%$, mycotic stomatitis $2 \%$, glossitis represented $37 \%$, glossodynia $41 \%$, periodontitis $38 \%$; 94 cases presented tartar, dental caries $34 \%$, parathyroid hypertrophy $6 \%$, angular cheilitis $34 \%$, pain syndrome $24 \%$.

The extensive and total edentations were frequently encountered as well as the atrophy of the alveolar ridges due to the loss of teeth through periodontal diseases.

34 cases $(20.62 \%)$ presented obesity. Oro-dental disorders were: sialorrhea 11 cases $(6.66 \%)$, hyperorexia 9 cases $(5.45 \%)$, glossitis 13 cases $(7.87 \%)$.

The patients with acute gout include 29 cases with acute gout in the chronic stage. They presented joint manifestations - 21 cases (12.72\%), acute gout attack - 4 cases $(2.42 \%)$, oral form - 2 cases $12.12 \%)$, uric nephropathy-2 cases (12.12\%).

Oral syndrome consisted of hyposalivation and xerostomia in the acute phase of gout with multiple caries. One of the cases had a buccal debut represented by temporo-mandibular arthritis, xerostomia, pharyngitis, dysphagia, red oral mucosa, difficulty in swallowing. The teeth are easily crushed. The abrasion is very early, sometimes the submaxillary glands are enlarged. 11 cases (7, 33\%) had gouty arthritis presenting joint attacks.

During the algic phases, the patients had salivary hypersecretion, xerostomia, hyperesthesia and dental 
neuralgia, increased salivary viscosity, with sodium urate crystals in the saliva.

The tempo-mandibular joint was very painful, with tendency to trimus (4 cases - 3.03\%).

Chronic marginal superficial periodonopathy is found characterized by jaw bone tension, papillary swelling, dental mobility, serous secretion in the pockets $[27,28]$.

There were 14 patients (8.48\%) with erythematous stomatitis and non-painful hyperkeratosis plates located in the gingival-jugal ditch.

The lesions in the oral cavity must be seen in the light of systemic pathological processes and correlated with systemic illnesses because systemic diseases produce oral manifestations and vice versa, oral disorders produce systemic manifestations.

\section{Conclusions}

Considering the human organism as a unitary whole and considering the increased frequency of oral manifestations during the onset and evolution of general disorders, the practical significance of oral pathological manifestations in the diagnosis, prognosis and therapeutic attitude in internal diseases results.

General pathology influences and is, in turn, influenced by oral pathology. Oral manifestations can hold the primary role in establishing early diagnosis in a serious condition that can be improved by appropriate therapy.

\section{References}

1.***ADA Standards of Medical Care in Diabetes - 2013. Diabetes Care. 2013;36(1):S11-66

2.***American Diabetes Association Diagnosis and classification of diabetes mellitus. Diabetes Care. 2013;36:S67-S74

3.***American Diabetes Association Diagnosis and classification of diabetes mellitus (Position Statement). Diabetes Care 2009;32(1):S62-S67

4.***American Diabetes Association, Bantle JP, Wylie-Rosett J, Albright $A L$, et al. Nutrition recommendations and interventions for diabetes: a position statement of the American Diabetes Association. Diabetes Care. 2008:31(1):S61-78

5.***Canadian Diabetes Association Clinical Practice Guidelines Expert Committee. Canadian Diabetes Association 2008 clinical practice guidelines for the prevention and management of diabetes in Canada. Can J Diabetes. 2008; 32 (1):S168-S180.

6.***Diabetes Prevention Program Research Group. The 10-year costeffectiveness of lifestyle intervention or metformin for diabetes prevention: an intent-to-treat analysis of the DPP/DPPOS. Diabetes Care. 2012;35:723-730.

7.HAMMAN RF, WING RR, EDELSTEIN SL. Effect of weight loss with lifestyle intervention on risk of diabetes. Diabetes Care. 2006;29:21022107.

8.HANCU N, NITA C, CRACIUN. Abecedar de Nutritie, 2012. Editura Sanatatea Press Group

9.BOTNARIU, G., POPA, A., MITREA, G., MANOLE, M., PACURAR, M., ANGHELE, M., CURIS, C., TEODORESCU, E., Correlation of Glycemic and Lipid Control Parameters with Cognitive Dysfunction Scores, in Type 2 Diabetic Persons Results from a cross- sectional study. Rev Chim (Bucharest), 2018, 69(12):3486-3489

10.CUCIUREANU, D.I., STATESCU, C., SASCAU, R.A., etal. Particularities of using contrast agents in diagnosis of stroke. Rev Chim (Bucharest), 70, no.2, 2019, p.685-688.

11.*** IDF Clinical Guidelines Task Force. Global Guideline on Pregnancy and Diabetes. Brussels: International Diabetes Federation 2010.
12.*** International Association of Diabetes and Pregnancy Study Groups Consensus Panel, Metzger BE, Gabbe SG, Persson B, et al. International association of diabetes and pregnancy study groups recommendations on the diagnosis and classification of hyperglycemia in pregnancy. Diabetes Care. 2010;33(3):676-82.

13.MINDRUTA IR, BAJENARU OA, PANEA CA, et al. Experience with lacosamide in treating focal epilepsy patients in Romania: efficacy, safety and time to reach response [abstract no. p332]. Epilepsia. 2014;55(Suppl 2):110

14.CUCIUREANU DI, NITA A, CUCIUREANU A, CUCIUREANU T , Constantinescu IM. Experience with first episode of consciousness loss assessment in a regional center of Romania. [abstract no. p638]. Epilepsia. 2016; 57 (suppl. 2): 194

15.J ARVELA IY, JUUTINEN J, KOSKELA P, et al. Gestational diabetes idenitifies women at risk for permanent type 1 and type 2 diabetes in fertile age. Diabetes Care. 2006;29:607-612.

16.KIM C, HERMAN WH, CHEUNG NW, etal. Comparison of hemoglobin Alc with fasting plasma glucose and 2-h postchallenge glucose for risk stratification among women with recent gestational diabetes mellitus. Diabetes Care. 2011; 34:1949-1951

17.KNOWLER WC, FOWLER SE, HAMMAN RF et al. Diabetes Prevention Program Re- search Group. 10-year follow-up of diabetes incidence and weight loss in the Diabetes Prevention Program Outcomes Study. Lancet. 2009;374:1677-1686.

18.RAFTU, G., MITREA, G., MACOVEI, LA., NECHITA, A., Chemical Additives from the Composition of Plastic Products and Other Materials in Establishing Diagnosis for Alergy Disease. Mat.Plast.,55, no.4, 2018, p.609-612

19.LANGER O. A spectrum of glucose thresholds may effectively prevent complications in the pregnant diabetic patient. Semin Perinatol. 2002:26:196-205.

20.BOTEZATU, C, DUCEAC, LD, MASTALIER, B, STAFIE, L, J ITAREANU, CM, LUCA, AC, TARCA, E, MITREA, G, IORDACHE, AC, PATRASCU, T. Hepatic cystic echinococcosis studied in a family group. INTERNATIONAL JOURNAL OF MEDICAL DENTISTRY, 2018, 22 (4): 346-350

21.LI G, ZHANG P, WANG J et al. The long-term effect of lifestyle interventions to prevent diabetes in the China Da Qing Diabetes Prevention Study:a 20-year follow-up study. Lancet. 2008;371:1783-1789. 22.LINDSTROM J, ILANNE-PARIKKA P, PELTONEN $M$ et al. Finnish Diabetes Prevention Study Group. Sustained reduction in the incidence of type 2 diabetes by lifestyle intervention: follow-up of the Finnish Diabetes Prevention Study. Lancet. 2006;368:1673-1679.

23.LONG S, NELMS M, SUCHER K. Nutrition therapy and pathophysiology. Belmont, CA:Thomas Wadsworth, 2007.

24.METZGER BE, GABBE SG, PERSSON B etal. International Association of Diabetes and Pregnancy Study Groups Consensus Panel. International association of diabetes and pregnancy study groups recommendations on the diagnosis and classification of hyperglycemia in pregnancy. Diabetes Care. 2010; 33:676-682.

25.MOTA M, POPA SG. Diabetul zaharat. In Patologia nutritional metabolica. Editat de Mota M., Dinca M. Editura Medicala Universitara Craiova. 2010:147-257. ISBN 978-973-106-154-2.

26.MULHOLLAND C, NJOROGE T, MERSEREAU P, et al. Comparison of guidelines available in the United States for diagnosis and management of diabetes before, during, and after pregnancy.J Womens Health (Larchmt). 2007;16(6):790-801

27.***World Health Organization, International Diabetes Federation. Definition and diagnosis of diabetes mellitus and intermediate hyperglycemia: report of a WHO/IDF consultation 2006; http:// www.who.int/ (last accessed 28 J anuary 2013).

28.WUCHER H, LEPERCQ J, TIMSIT J. Onset of autoimmune type 1 diabetes during pregnancy:Prevalence and outcomes. Best Pract Res Clin Endocrinol Metab. 2010;24(4):617-24. 